\title{
Hybrid Super-Nyquist CAP Modulation based VLC with Low Bandwidth Polymer LEDs
}

\author{
Paul Anthony Haigh ${ }^{1,2}$, Petr Chvojka ${ }^{3}$, Alessandro Minotto ${ }^{4,5}$, Andrew Burton ${ }^{6}$, Petri Murto ${ }^{7}$, \\ Ergang Wang ${ }^{7}$, Zabih Ghassemlooy ${ }^{6}$, Stanislav Zvanovec ${ }^{3}$, Franco Cacialli ${ }^{4,5}$ and Izzat Darwazeh $^{2}$ \\ ${ }^{1}$ Intelligent Sensing and Communications Group, Newcastle University, UK \\ ${ }^{2}$ Information and Communications Engineering Group, University College London, UK \\ ${ }^{3}$ Department of Electromagnetic Field, Czech Technical University in Prague, Czech Republic \\ ${ }^{4}$ Department of Physics and Astronomy, University College London, UK \\ ${ }^{5}$ London Centre for Nanotechnology, University College London, UK \\ ${ }^{6}$ Optical Communications Research Group, Northumbria University, UK \\ ${ }^{7}$ Department of Chemistry and Chemical Engineering/Applied Chemistry, \\ Chalmers University of Technology, Sweden \\ paul.haigh@newcastle.ac.uk
}

\begin{abstract}
Visible light communication systems often suffer from high frequency attenuation when transmitting outof-band. This effect has been ameliorated by multi-band modulations such as multi-band carrier-less amplitude and phase ( $m$-CAP), which minimises the effect of decreased high frequency magnitude and maximises signal-to-noise ratio-per-sub-band. On the other hand, in the pass-band region, super-Nyquist CAP (SCAP) can offer throughput improvements with no additional complexity at the receiver, at the cost of bit error rate. We propose, for the first time, a new hybrid SCAP modulation format that takes advantageous of both SCAP (i.e. overlapped sub-bands within the modulation bandwidth) and conventional $m$ CAP (orthogonally spaced bands outside the modulation bandwidth) while maintaining isolation between noise sources. We show higher baud rates within the passband region whilst supporting out-of-band transmission at lower error vector magnitudes.
\end{abstract}

Index Terms-Carrier-less amplitude and phase modulation, digital signal processing, modulation, visible light communications

\section{INTRODUCTION}

Polymer light-emitting diodes (PLEDs) represent an attractive alternative to conventional LEDs as the transmitter in visible light communications (VLC). PLEDbased screens in smart devices can be used for $(i)$ the uplink, a challenge in VLC where infrared [1] or radio frequencies [2] have typically been proposed; and (ii) embedding information onto the displayed image, thus allowing users to use the device as desired whilst still maintaining an active link. In our previous work [3], we showed that using organic PLEDs in a smart-display consisting of individual red-green-blue (RGB) pixels

PAH and PC contributed equally. This project is supported by the UK EPSRC grant EP/P006280/1: MARVEL, the Czech Science Foundation project GACR 17-17538S and the Horizon 2020 Framework Programme MSC ITN grant 764461 (VISION). FC is a Royal Society Wolfson Research Merit Award recipient. EW acknowledges the Swedish Research Council, Swedish Research Council Formas and the Wallenberg Foundation. capable of transmitting independent information could yield an advantage for a VLC uplink with an aggregated data rate of $55 \mathrm{Mb} / \mathrm{s}$, which is generally sufficient for indoor applications.

However, one of the main problems with PLEDs is their inherently lower modulation bandwidth (typically in the order of hundreds of $\mathrm{kHz}$ ) due to limited charge transport characteristics and large device area, which combined with sub-micron thickness result in high plate capacitances. This is a serious challenge for high-speed PLED-based VLC systems. In the literature, the vast majority of studies reported are focused on using pulseamplitude modulation (PAM) with time-domain equalisation to overcome low bandwidth and increase speeds from several $\mathrm{kb} / \mathrm{s}$ to $55 \mathrm{Mb} / \mathrm{s}[3,4]$.

Alternatively, subcarrier modulation formats with high spectral efficiency such as orthogonal frequency division multiplexing (OFDM) [5], carrier-less amplitude and phase (CAP) modulation or multi-band CAP $(m-$ CAP) [6], where $m$ is the number of sub-bands (SBs), have been investigated to increase data rates. In [7], a comparative study of OFDM and CAP performed over an RGB-LED-based VLC link was carried out showing that 1-CAP $(3.22 \mathrm{~Gb} / \mathrm{s})$ outperformed OFDM $(2.93 \mathrm{~Gb} / \mathrm{s})$ in terms of data rate and bit-error rate (BER) performance. In $[8,9]$, it was shown that both the data rate and spectral efficiency improves with increasing $m$.

Recently, non-orthogonal modulation techniques such as spectrally efficient frequency division multiplexing (SEFDM) [10] and faster-than-Nyquist (FTN) signalling [11] have been investigated in VLC systems. These modulation formats are intended to improve the link capacity by intentionally introducing inter-carrier interference (ICI) or inter-symbol interference (ISI) in SEFDM and FTN, respectively. Note, the introduction of interference enables transmission of more symbols-per-second in the 
same time period compared to conventional orthogonal systems. In [12], a non-orthogonal variation on $m$-CAP was proposed called super-Nyquist $m$-CAP ( $m$-SCAP), where SBs are purposely overlapped reducing the total signal bandwidth and causing inter-band interference (IBI). Hence, higher symbol rates can be supported at the cost of BER. It was shown that 10-SCAP offered spectral efficiency improvement by $\sim 45 \%$ with no additional digital signal processing at the receiver.

Therefore, considering the inherent low modulation bandwidth of PLEDs, $m$-SCAP could be an attractive modulation format for achieving a greater capacity. On the other hand, in VLC the signal bandwidth is generally higher than the modulation bandwidth, thus resulting in significant high frequency attenuation [13] i.e. higher BER. Hence, in this work, we propose and experimentally demonstrate a novel hybrid Super-Nyquist CAP modulation format including both super-Nyquist and orthogonal $m$-CAP, where the SBs placed in the pass- and stop-bands of the PLED frequency response are non-orthogonal and orthogonal, respectively. This is intended to separate the two dominant factors of IBI and high frequency attenuation in the super-Nyquist and orthogonal cases, respectively.

The rest of the paper is organised as follows; in Section II the proposed hybrid scheme is theoretically formulated and details of the fabrication and characterisation of the PLED under test as well as the VLC link details are provided. In Section III, measurement results are discussed and in Section IV conclusions are drawn.

\section{HYBRID SUPER-NYQUIST CAP AND PLED}

\section{A. Hybrid Multi-Band CAP Modulator}

The concept of hybrid super-Nyquist $m$-CAP ( $m$ HSCAP) is shown in terms of the normalised magnitude responses of the transmit filters in Fig. 1 with 4 SBs, where the $1^{\text {st }}$ two SBs are overlapped and $2^{\text {nd }}$ two are orthogonally spaced. We selected a total transmission bandwidth of $1 \mathrm{MHz}$ (i.e., the $1^{\text {st }}$ two super-Nyquist SBs are located in the pass-band of the PLED frequency response $H(f)$ with a 3-dB modulation bandwidth of $500 \mathrm{kHz}$. Therefore, the $1^{\text {st }}$ two SBs have a bandwidth of $312.5 \mathrm{kHz}$ each in comparison to the $250 \mathrm{kHz}$ bandwidth occupied by the $2^{\text {nd }}$ two orthogonal SBs. Note, beyond the cut-off frequency the attenuation significantly increases $(\sim 10 \mathrm{~dB} /$ decade $)$ due to the frequency response $H(f)$ of the PLED, see Fig. 1, reportedly following a $1^{\text {st }}$ order low-pass response [3].

The $m$-HSCAP signal is generated following the block diagram in Fig. 2. At the transmitter, the super-Nyquist and orthogonal SBs are generated as two separate entities. First, $m$ independent data streams from $\mathbf{D}_{\mathbf{1}}$ to $\mathbf{D}_{\mathbf{m}}$ are generated, which are then mapped onto the 4-level quadrature amplitude modulation (QAM) constellation before being split into their in-phase $(s(t))$ and quadrature components $(\bar{s}(t)))$, followed by up-sampling via

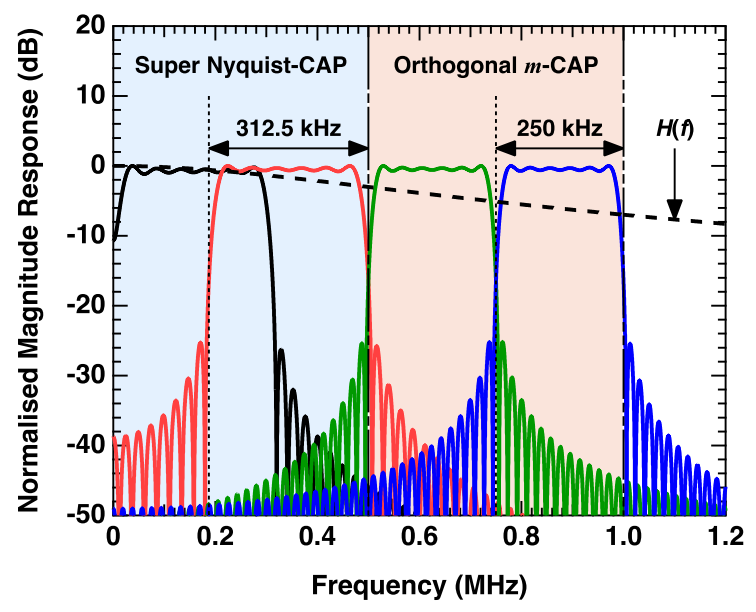

Fig. 1. Conceptual frequency response of the proposed hybrid modulation format

zero-padding. The total baud rate for the super-Nyquist $R_{s N}$ system is given by:

$$
R_{s N}=\frac{B_{N}}{(1+\beta)(1-\alpha)}
$$

where $B_{N}$ is the portion of the signal bandwidth $B_{s i g}$ dedicated to the non-orthogonal SBs, $\beta$ is the excess bandwidth factor of the pulse shaping filters and $\alpha$ is the bandwidth expansion factor, which controls the increase in $R_{s N}$. For the orthogonal part, the symbol rate $R_{S O}$ is given by:

$$
R_{s O}=\frac{B_{s i g}-B_{N}}{1+\beta}
$$

Note, the number of samples-per-symbol $N_{S S}$ controls the sampling frequency $f_{s}$ for each part of the system. The nature of $m$-HSCAP, where two different symbol rates are essentially being transmitted, means that the scheme must be multi-rate until the point of SB aggregation (see the modulator in Fig. 2). In both cases, for up-sampling we have [6]:

$$
N_{S S}=\gamma\lceil 2 m(1+\beta)\rceil
$$

where $\gamma$ is an over-sampling factor set to 4 in this work without any loss of generality.

After up-sampling, the data streams are then passed through the pulse shaping filters, usually given as the product of a square-root raised cosine (SRRC) $g(t)$ with excess bandwidth $\beta$ and a cosine/sine wave pair [14]. The $i^{\text {th }}$ in-phase $p_{i}(t)$ and imaginary $\overline{p_{i}}(t)$ pulse shaping filter for either entity are [14]:

$$
\begin{aligned}
& p_{i}(t)=g(t) \cos \left(2 \pi f_{i} t\right) \\
& \bar{p}_{i}(t)=g(t) \sin \left(2 \pi f_{i} t\right)
\end{aligned}
$$




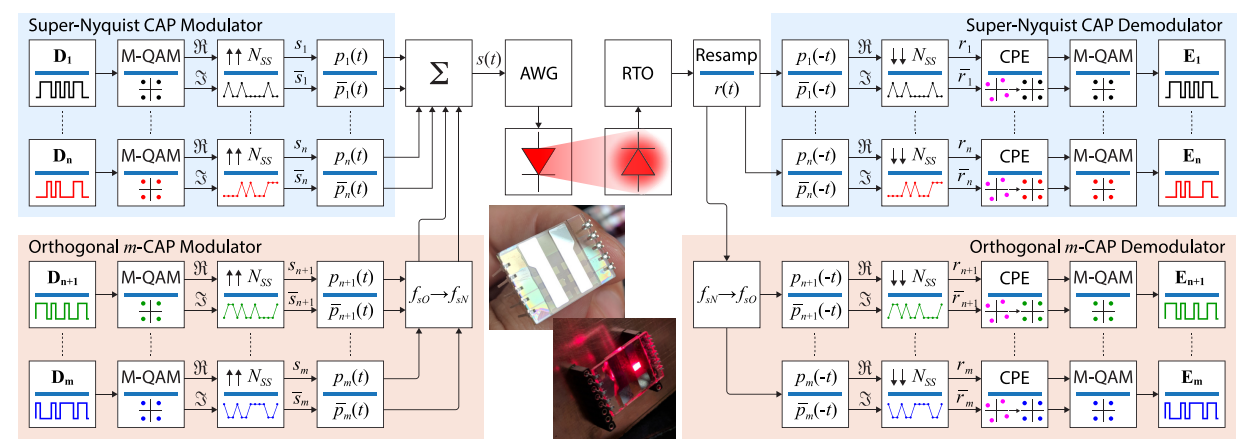

Fig. 2. Simplified block diagram for the proposed $m$-HSCAP scheme; inset shows two PLEDs used

where $f_{i}$ is the carrier frequency of $i^{\text {th }}$ sub-carrier given as:

$$
f_{i}= \begin{cases}\frac{B_{N \text { min }}}{2}+\frac{i\left(1-B_{N \min }\right)}{n-1} & i \in\{1, \ldots, n\} \\ B_{N}+\frac{B_{\min }(2 i-1)}{2} & i \in\{n+1, \ldots, m\}\end{cases}
$$

where $n$ is the number of SBs assigned to the superNyquist part, $B_{N \min }$ is the minimum bandwidth occupied by a single non-orthogonal SB with $R_{s N}$ and $B_{\text {min }}$ is the bandwidth occupied by an orthogonal SB with $R_{s O}$. Note, the mathematical details on pulse shapes are widely documented [14].

As mentioned, at the point of pulse shape filtering the system is multi-rate for any value of $\alpha>0$. Thus, two different relationships define the the minimum sampling frequency that satisfies the Nyquist rate. For the superNyquist part, the minimum sampling frequency is given by:

$$
f_{s N}=\frac{R_{s N} N_{S S}}{n}
$$

while the orthogonal part is given by:

$$
f_{s O}=\frac{R_{s O} N_{S S}}{m-n}
$$

Clearly, $f_{s N}>f_{s O}$ is a constant condition for this scheme except for the special case of no expansion (i.e. $\alpha=0$ ), where $f_{s N}=f_{s O}$. Therefore, it is necessary to resample the orthogonal entity from $f_{s O} \longrightarrow f_{s N}$ before summation, where each sample must be transmitted at the same rate. After resampling, the two entities are directly summed for transmission as [6,14]:

$$
s(t)=\sum_{i=1}^{m}\left[s_{i}(t) \otimes p_{i}(t)-\overline{s_{i}}(t) \otimes \overline{p_{i}}(t)\right]
$$

which results in the superposed spectrum, see Fig. 3 . Note the $\sim 6 \mathrm{~dB}$ power penalty, which is dependent on both $n$ and $\beta$ as reported in [12].

The entire physical setup is controlled and automated using LabVIEW to ensure equivalent test conditions for all signals under test. The generated signal $s(t)$ is then loaded into a Tektronix AWG70002A arbitrary waveform generator (AWG), with $4 \mathrm{GHz}$ analogue bandwidth and 10-bit vertical resolution, the output of which is used for intensity modulation of PLED via a $4 \times$ amplifier (TI THS3202) and a custom-built driver which supplies $20 \mathrm{~mA} \mathrm{dc}$ and $20 \mathrm{~mA}$ ac currents.

\section{B. PLED Fabrication and Characterisation}

PLEDs used here were fabricated using oxygen plasma treated indium tin oxide (ITO) substrates as transparent anodes (OSSILA Ltd) [15]. On top of these, a $40 \mathrm{~nm}$ poly(3,4-ethylenedioxythiophene)poly(styrenesulfonate) (PEDOT:PSS) hole-injection layer was deposited via spin coating from a water dispersion (Heraeus Clevios AI 4083) in air and annealed at $150^{\circ} \mathrm{C}$ for 10 minutes in a nitrogenfilled glove-box. Following this, the active polymer (PIDT-2TPD, number average molecular weight = $63.7 \mathrm{kDa}$, polydispersity index $=2.3$ ), whose chemical structure and synthesis were reported in [16,17], was deposited via spin-coating from a toluene solution, following the same protocol used in [18]. Finally, a calcium/aluminium $(\mathrm{Ca} / \mathrm{Al}$ with thicknesses of 30 and $200 \mathrm{~nm}$, respectively) cathode was thermally evaporated on top, to obtain, for each substrate, six independent

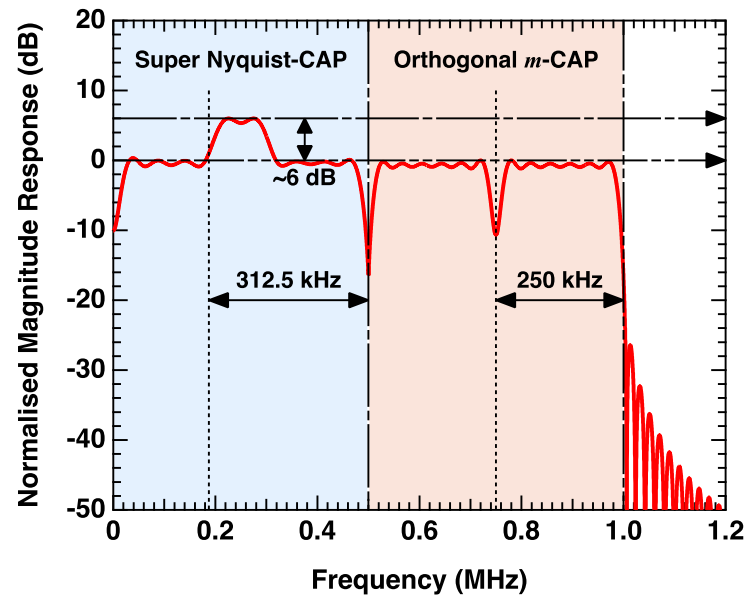

Fig. 3. Superposed ideal magnitude response of the proposed hybrid scheme 
pixels with an active area of $4.5 \mathrm{~mm}^{2}$, as defined by the overlap between the patterns of the ITO anode and the $\mathrm{Ca} / \mathrm{Al}$ cathode. To prevent oxidation of the $\mathrm{Ca}$ and the emissive polymer layers, therefore preserving the device stability when operated in air, each PLED was encapsulated by covering the active area and metal electrodes with a layer of epoxy resin (OSSILA Ltd) and a protective glass slide. The resin was then cured by exposing the PLEDs to UV light for 15 minutes in the glovebox, see photos inset in Fig. 2.

The current-voltage $(I-V)$ and current-optical power density $(I-L)$ relationships were measured using a Keithley 2400 source-meter for dc power supply and a calibrated photodetector and are illustrated as an $L$ $I$ - $V$ curve in Fig. 4, which also shows the operating conditions for intensity modulation. The PLED has a clear pseudo-linear operating region over the ac current range selected, as highlighted by the linear fit $L_{\mathrm{fit}}=330.72 I_{\mathrm{dc}}-1.5698$, where $I_{\mathrm{dc}}(\mathrm{A})$ is the drive current. The emission spectrum is depicted inset in Fig. 4 showing strong red emission with a peak at $639 \mathrm{~nm}$ and two shoulders in the near-infrared region with peaks at $\sim 688 \mathrm{~nm}$ and $\sim 760 \mathrm{~nm}$ originating from weaker vibronic transitions. A single $4.5 \mathrm{~mm}^{2}$ PLED pixel and a link distance of $5 \mathrm{~cm}$ are adopted for consistency with previous work $[3,19]$ and to avoid excessive power burden on the device. The measured PLED bandwidth is $\sim 500 \mathrm{kHz}$ as illustrated by $H(f)$ in Fig. 1 and [19], which shows the rise time equivalent.

\section{Hybrid Multi-Band CAP Demodulator}

At the receiver, a photodetector (Thorlabs PDA36A2, composed of a $12 \mathrm{MHz}$ photodiode and transimpedance amplifier) and a $20 \mathrm{~dB}$ amplifier are used to regenerate the electrical signal, which is captured using an $8 \mathrm{GHz}$ Tektronix MSO70804C real time oscilloscope (RTO) at $62.5 \mathrm{MS} / \mathrm{s}$.

Next, the sampled signals are processed in MATLAB beginning with resampling to the original transmit rate

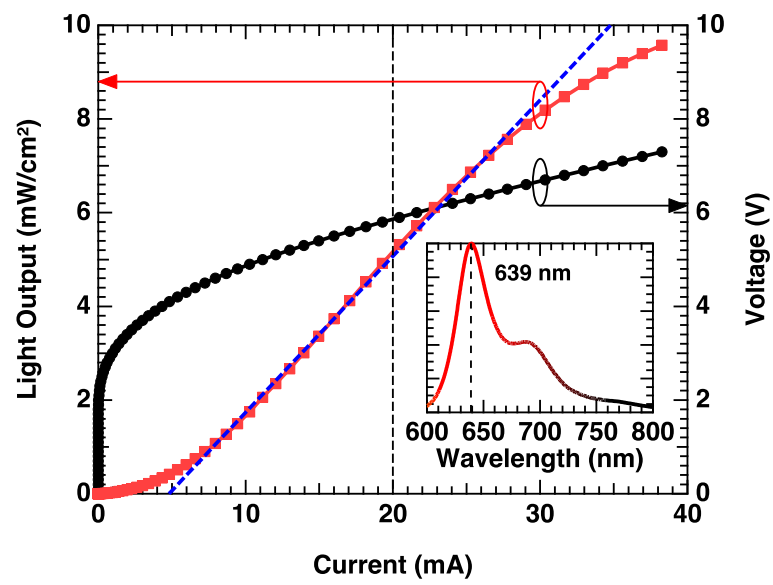

Fig. 4. Measured $L-I-V$ curve of the PLED under test. Inset: measured electroluminescence spectrum measured at the operating point of $f_{s N}$. The signals are then passed through a $4^{\text {th }}$ order low-pass filter (not shown in Fig. 2) to remove outof-band noise. This is followed by splitting the signal into their original super-Nyquist and orthogonal entities. For super-Nyquist, the signal is passed through a timereversed matched filter pair, which effectively band-pass filters the in-phase and quadrature components for each $\mathrm{SB}$, before down-sampling at a rate of $N_{S S}$. Next, the common phase error (CPE) is removed using conventional techniques reported widely in [20] before constellation de-mapping and estimation of the transmitted bits $\mathbf{E}_{\mathbf{i}}$ for the $i^{\text {th }}$ channel. For orthogonal SBs, the process is identical except for an additional resampling performed before matched filtering to recover the original sampling rate of the signal before transmission, recalling that $f_{s O} \neq f_{s N}$ when $\alpha>0$. A minimum of $10^{-6}$ bits are captured for each SB and the root mean square error vector magnitude $\left(\mathrm{EVM}_{\mathrm{RMS}}\right)$ is measured for every SB.

\section{RESULTS}

The measured $\mathrm{EVM}_{\mathrm{RMS}}$ of $m$-HSCAP for $\beta=0.5$ is illustrated in Fig. 5 for $m=\{10,8,6$ and 4\}. In each case, half of the SBs exist in the non-orthogonal region (dashed lines) and the rest in the orthogonal region (solid lines) of the signal bandwidth. As shown in Fig. 5 it is clear in every case that, orthogonal signals in the stopband of $H(f)$ can be supported with an $\mathrm{EVM}_{\mathrm{RMS}}$ in the range of $10-20 \%$ with a loose dependence on the number of SBs used. This is counter intuitive as it is expected, based on the literature [8], that the $\mathrm{EVM}_{\mathrm{RMS}}$ decreases with increasing $m$. Therefore, we attribute this to the increased peak-to-average power ratio (PAPR) associated with higher orders of $m$, thus causing a limitation on the received signal-to-noise ratio [21]. Furthermore, the $\mathrm{EVM}_{\mathrm{RMS}}$ increases with the carrier frequencies of the
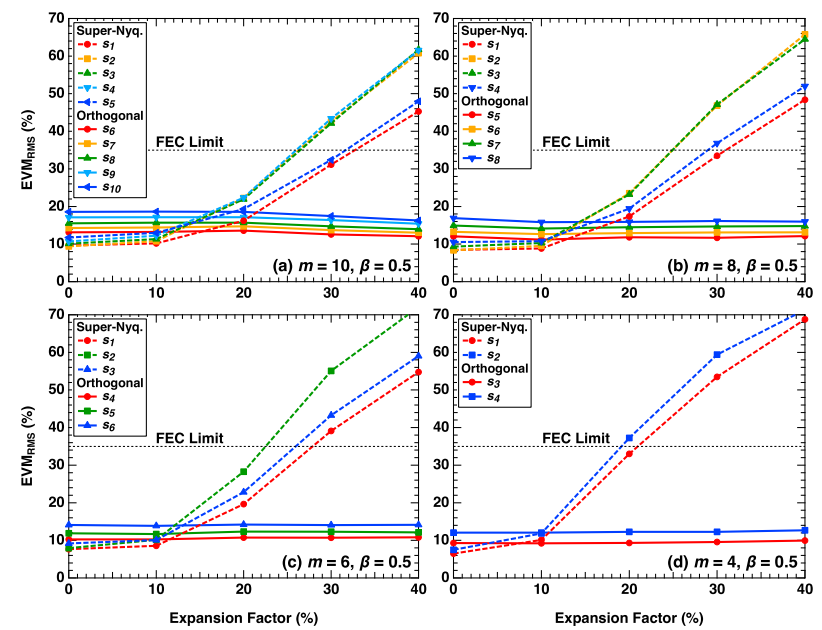

Fig. 5. $E \mathrm{VM}_{\mathrm{RMS}}$ measurements for both the orthogonal (solid lines) and super-Nyquist (dashed) for the $s_{i}{ }^{\text {th }}$ sub-band, $\beta=0.5$ and (a) $m=10$, (b) $m=8$, (c) $m=6$ and (d) $m=4$ 
orthogonal SB, due to the increased attenuation experienced.

In terms of the non-orthogonal SBs located inside the PLED modulation bandwidth, it is clear that EVM $_{\mathrm{RMS}}$ experienced for a higher number of sub-bands is lower, i.e. for $\alpha=20 \%$, the average $\mathrm{EVM}_{\mathrm{RMS}}$ values are $\sim 20 \%$ and $35 \%$ for $m=10$ and 4 , respectively. This is because of a combination of the reduced SB bandwidth and increased excess energy (i.e. $\beta=0.5$ ) in the pulseshaping filters. Note, increasing $m$ results in reduced relative overlap between the super-Nyquist SBs [12]. Hence, when combined with the additional excess energy provided for high $\beta$, IBI is reduced and therefore highorder systems can tolerate an increased level of expansion at lower values of $\mathrm{EVM}_{\mathrm{RMS}}$. This is supported by the fact that for higher values of $m$ the $\mathrm{EVM}_{\mathrm{RMS}}$ drops well below the threshold level of $\sim 35 \%$ (corresponding to a $7 \%$ forward error correction (FEC) BER limit of $3.8 \times 10-3)$.

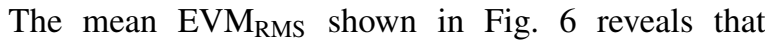
higher expansion can be supported with aggregate error rates below the FEC limit, and in principle should be supported by up to $\sim 25 \%$ for $m \geq 6$. This agrees with literature reported on super-Nyquist CAP systems where up to $30 \%$ of bandwidth can be saved when used in a compressive mode, i.e. where the overall signal bandwidth is reduced, and the baud rate is not increased [12]. It is also indicated that limited improvement is achieved by increasing the number of SBs since there is limited improvement in $\mathrm{EVM}_{\mathrm{RMS}}$ for $m>8$. This is also in good agreement with the literature, which shows the benefits of $m$-CAP plateau beyond a given number of sub-bands depending on the system being adopted $[8,22]$.

In terms of overall throughput, for $m$-HSCAP the total bit rate $R_{b T} 1.5 \mathrm{Mb} / \mathrm{s}$ (using (1) and (2)) for $\beta=0.5$, $\alpha=20 \%$ and 4-QAM (and $m>4$ ). Under the same

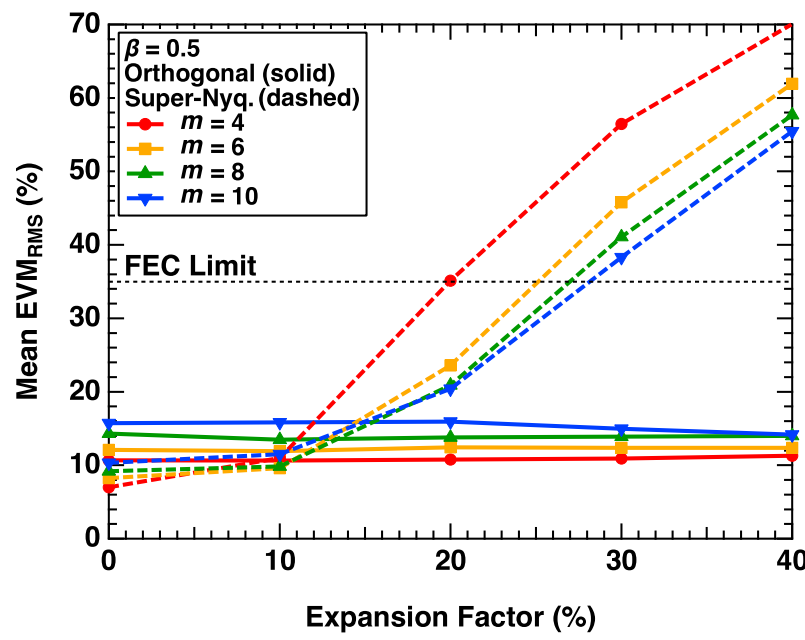

Fig. 6. Mean $\mathrm{EVM}_{\mathrm{RMS}}$ dependance on the expansion factor $(\beta=0.5)$ conditions, $m$-HSCAP offers throughput improvements of $\sim 67 \%$ and $12 \%$ compared to $m$-SCAP (with $R_{s N}$ of $\sim 450 \mathrm{kbaud}(900 \mathrm{~kb} / \mathrm{s})$ and $B_{\bmod }$ of $\left.500 \mathrm{kHz}\right)$ and $m$ CAP (with $R_{s T}$ of $\sim 667 \mathrm{kbaud}(1333 \mathrm{~kb} / \mathrm{s})$ and $B_{s i g}$ of $1 \mathrm{MHz}$ ), respectively. Similar analysis is performed for the same system but for lower energy levels in the guard band, i.e., $\beta=0 / 1$. The full $\mathrm{EVM}_{\mathrm{RMS}}$ results as a function of the expansion factor are presented in Fig. 7. For the orthogonal case, the EVM $\mathrm{RMS}_{\mathrm{R}}$ levels are almost constant for the range of $m$, but are reduced by an average of $4 \%$ compared to Fig. 5. The reason for this is that lower $\beta$ requires a higher number of filter-taps to achieve the equivalent $\mathrm{EVM}_{\mathrm{RMS}}$ (or BER) [14]. In this work, we kept the number of filter-taps constant to maintain a constant computational load, as is the general case in $m$-CAP with different values of $\beta[12,14]$. For the super-Nyquist SBs with a similar trend as in Fig. 5, albeit at a lower $\alpha$ than previously experienced, the EVM $\mathrm{RMS}_{\text {s }}$ values below the FEC limit are within the expansion range of $10-15 \%$ compared to $20-30 \%$ in Fig. 5. This is because of overlapping SBs, due to the reduced excess energy (i.e., lower $\beta$ ), where IBI induced degradation on the signal constellation prior to demodulation is more significant, thus resulting in higher BERs.

Finally, in Fig. 8 the measured mean EVM RMS $_{\text {against }}$ the expansion factor for a range of $m$ and $\beta$ of 0.1 is illustrated. The variation between measurements is clearly reduced in comparison to Fig. 6. Note from observation of Figs. 6 and . 8, $\beta$ is the key parameter in $m$-HSCAP, which influences the achievable $\alpha$ and the number of SBs that can be used in the superNyquist case. In addition, clearly a higher rate, which is proportional to the sum of (1) and (2), can be achieved with a larger number of SBs (i.e. up to $m=10$ ) for
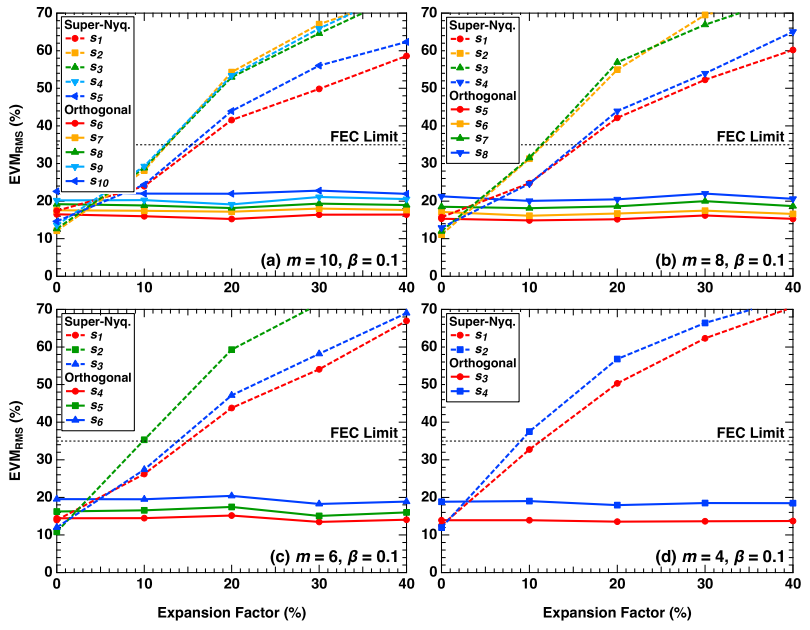

Fig. 7. $E \mathrm{VM}_{\mathrm{RMS}}$ measurements for both the orthogonal (solid lines) and super-Nyquist (dashed) for the $s_{i}{ }^{\text {th }}$ sub-band, $\beta=0.1$ and (a) $m=10$, (b) $m=8$, (c) $m=6$ and (d) $m=4$ 


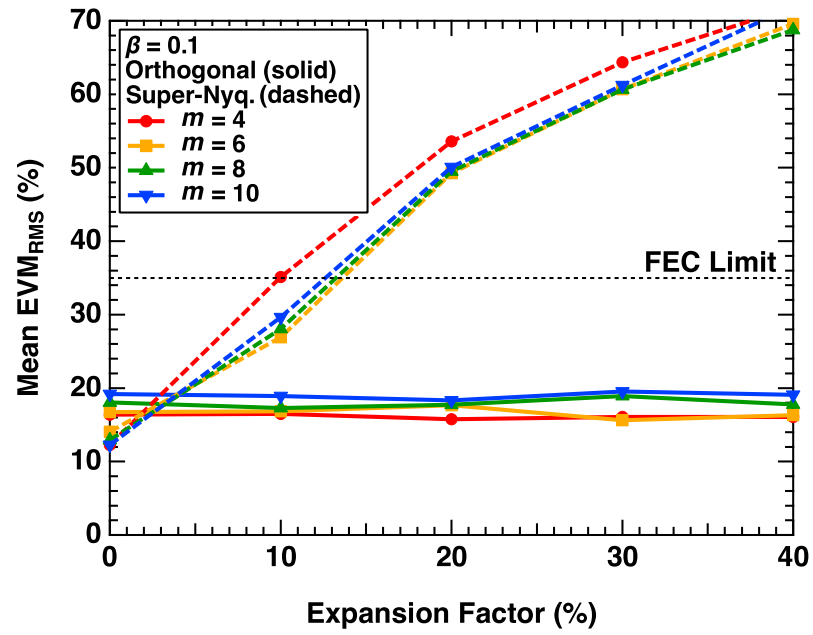

Fig. 8. Mean $\mathrm{EVM}_{\mathrm{RMS}}$ dependence on the expansion factor $(\beta=0.1)$

$\beta=0.5$, since a higher $\alpha$ can be reached considering the mean $\mathrm{EVM}_{\mathrm{RMS}}$. However, for $\beta=0.1$ (i.e. Fig. 8), there is little difference between the case where $m=6$ to 10 . This could be used to advantageously design the signal to remove computational complexity via removing filters from the system structure altogether. In terms of throughput, $m$-HSCAP can support an overall bit rate of $1.92 \mathrm{Mb} / \mathrm{s}$, following (1) and (2) for $\beta=0.1, \alpha=10 \%$ and 4-QAM. Using the same method as previous, we calculated that $m$-SCAP and conventional $m$-CAP can deliver bit rates of $\sim 1 \mathrm{Mb} / \mathrm{s}$ and $1.8 \mathrm{Mb} / \mathrm{s}$, respectively which corresponds to improvements of $\sim 90 \%$ and $7 \%$, respectively.

\section{CONCLUSION}

In this work, we proposed a hybrid modulation format, which improved the throughput of both $m$-SCAP and conventional $m$-CAP systems by up to $90 \%$ and $7 \%$, respectively. We showed that for orthogonal signals in the stop-band of PLED the measured $\mathrm{EVM}_{\mathrm{RMS}}$ were in the range of $10-20 \%$ with a loose dependence on the number of SBs, whereas for the non-orthogonal signals located within pass-band of PLED the average EVMRMS were $\sim 20 \%$ and $35 \%$ for $m=10$ and 4 , respectively.

\section{REFERENCES}

[1] A. Burton, H. Le Minh, Z. Ghassemlooy et al., "Experimental demonstration of $50-\mathrm{Mb} / \mathrm{s}$ visible light communications using $4 \times 4$ MIMO," IEEE Photonics Technology Letters, vol. 26, no. 9, pp. 945-948, 2014.

[2] S. H. Shao, A. Khreishah, M. Ayyash et al., "Design and analysis of a visible-light-communication enhanced WiFi system," Journal of Optical Communications and Networking, vol. 7, no. 10, pp. 960973, 2015.

[3] P. A. Haigh, F. Bausi, H. Le Minh et al., "Wavelength-multiplexed polymer LEDs: Towards $55 \mathrm{Mb} / \mathrm{s}$ organic visible light communications," IEEE Journal on Selected Areas in Communications, vol. 33, no. 9, pp. 1819-1828, 2015.

[4] P. A. Haigh, F. Bausi, Z. Ghassemlooy et al., "Visible light communications: real time $10 \mathrm{Mb} / \mathrm{s}$ link with a low bandwidth polymer light-emitting diode," Opt Express, vol. 22, no. 3, pp. 2830-8, 2014.
[5] J. Wang, Y. Xu, X. Ling et al., "PAPR analysis for OFDM visible light communication," Optics express, vol. 24, no. 24, pp. 27457 $27474,2016$.

[6] M. I. Olmedo, T. J. Zuo, J. B. Jensen et al., "Multiband carrierless amplitude phase modulation for high capacity optical data links," Journal of Lightwave Technology, vol. 32, no. 4, pp. 798-804, 2014.

[7] F. M. Wu, C. T. Lin, C. C. Wei et al., "Performance comparison of OFDM signal and CAP signal over high capacity RGB-LED-Based WDM visible light communication," IEEE Photonics Journal, vol. 5, no. 4, pp. $7901507-7901507,2013$.

[8] P. A. Haigh, A. Burton, K. Werfli et al., "A multi-cap visiblelight communications system with $4.85-\mathrm{b} / \mathrm{s} / \mathrm{Hz}$ spectral efficiency," IEEE Journal on Selected Areas in Communications, vol. 33, no. 9, pp. 1771-1779, 2015.

[9] P. A. Haigh, S. T. Le, S. Zvanovec et al., "Multi-band carrier-less amplitude and phase modulation for bandlimited visible light communications systems," IEEE Wireless Communications, vol. 22, no. 2, pp. 46-53, April 2015.

[10] Y. Wang, Y. Zhou, T. Gui et al., "Efficient MMSE-SQRD-based MIMO decoder for SEFDM-based 2.4-Gb/s-spectrum-compressed WDM VLC system," IEEE Photonics Journal, vol. 8, no. 4, pp. $1-9,2016$.

[11] S. Liang, Z. Jiang, L. Qiao et al., "Faster-than-Nyquist precoded CAP modulation visible light communication system based on nonlinear weighted look-up table predistortion," IEEE Photonics Journal, vol. 10, no. 1, pp. 1-9, 2018.

[12] P. Haigh, P. Chvojka, Z. Ghassemlooy et al., "Visible light communications: multi-band super-Nyquist CAP modulation," Optics Express, vol. 27, no. 6, pp. 8912-8919, 2019.

[13] Y. Wang, X. Huang, L. Tao et al. " "4.5-Gb/s RGB-LED based WDM visible light communication system employing CAP modulation and RLS based adaptive equalization," Optics express, vol. 23, no. 10, pp. 13626-13633, 2015.

[14] P. Chvojka, K. Werfli, S. Zvanovec et al., "On the $m$-CAP performance with different pulse shaping filters parameters for visible light communications," IEEE Photonics Journal, vol. 9, no. 5, pp. $1-12,2017$.

[15] T. M. Brown, G. M. Lazzerini, L. J. Parrott et al., "Time dependence and freezing-in of the electrode oxygen plasma-induced work function enhancement in polymer semiconductor heterostructures," Organic Electronics, vol. 12, no. 4, pp. 623-633, 2011.

[16] A. Minotto, P. Murto, Z. Genene et al., "Efficient nearinfrared electroluminescence at $840 \mathrm{~nm}$ with "Metal-Free" SmallMolecule:Polymer Blends," Adv Mater, vol. 30, no. 34, p. e1706584, 2018

[17] P. Murto, Z. Genene, C. M. Benavides et al., "High performance all-polymer photodetector comprising a donor-acceptor-acceptor structured indacenodithiophene-bithieno [3, 4-c] pyrroletetrone copolymer," ACS Macro Letters, vol. 7, no. 4, pp. 395-400, 2018.

[18] A. Zampetti, A. Minotto, B. M. Squeo et al., "Highly efficient solid-state near-infrared organic light-emitting diodes incorporating ADA dyes based on $\alpha, \beta$-unsubstituted "BODIPY" moieties," Scientific reports, vol. 7, no. 1, p. 1611, 2017.

[19] P. A. Haigh, A. Minotto, A. Burton et al., "Experimental demonstration of staggered CAP modulation for low bandwidth redemitting polymer-LED based visible light communications," arXiv preprint arXiv:1234:56789, 2019

[20] S. T. Le, P. A. Haigh, A. D. Ellis et al., "Blind phase noise estimation for CO-OFDM transmissions," Journal of Lightwave Technology, vol. 34, no. 2, pp. 745-753, 2016.

[21] M.-A. Khalighi, S. Long, S. Bourennane et al., "PAM-and CAPbased transmission schemes for visible-light communications," IEEE Access, vol. 5, pp. 27 002-27 013, 2017.

[22] P. A. Haigh, E. Hugues-Salas, and J. Wei, "On the performance of increasing subcarrier order in multi-band carrier-less amplitude and phase modulation for short haul optical links," in Asia Communications and Photonics Conference. Optical Society of America, 2017, pp. S4B-6. 